\title{
Counting points of slope varieties over finite fields
}

\author{
Thomas Enkosky \\ Department of Mathematics \\ University of Kansas \\ Lawrence KS, U.S.A. \\ tenkosky@math.ku.edu
}

Submitted: Oct 10, 2010; Accepted: Dec 7, 2010; Published: Jan 5, 2011

Mathematics Subject Classification: 05C30, 14G15, 05A19

\begin{abstract}
The slope variety of a graph is an algebraic set whose points correspond to drawings of that graph. A complement-reducible graph (or cograph) is a graph without an induced four-vertex path. We construct a bijection between the zeroes of the slope variety of the complete graph on $n$ vertices over $\mathbb{F}_{2}$, and the complementreducible graphs on $n$ vertices.
\end{abstract}

\section{Introduction}

Fix a field $\mathbb{F}$ and a positive integer $n$. Let $P_{1}=\left(x_{1}, y_{1}\right), \ldots, P_{n}=\left(x_{n}, y_{n}\right)$ be points in the plane $\mathbb{F}^{2}$ such that the $x_{i}$ are distinct. Let $L_{1,2}, \ldots, L_{n-1, n}$ be the $\left(\begin{array}{l}n \\ 2\end{array}\right)$ lines in $\mathbb{F}^{2}$ where $L_{i, j}$ is the line through $P_{i}$ and $P_{j}$. The slope variety $S_{\mathbb{F}}\left(K_{n}\right)$ is the set of possible $\left(\begin{array}{l}n \\ 2\end{array}\right)$-tuples $\left(m_{1,2}, \ldots, m_{n-1, n}\right)$, where $m_{i, j}=\frac{y_{i}-y_{j}}{x_{i}-x_{j}}$ denotes the slope of $L_{i, j}$ (if $\mathbb{F}$ is a finite field with $q$ elements then we use the notation $S_{q}\left(K_{n}\right)$ ). Over an algebraically closed field, the slope variety is the set of simultaneous solutions of certain polynomials $\tau_{W}$, called tree polynomials $[4,5]$, indexed by wheel subgraphs of the complete graph $K_{n}$. (A $k$-wheel is a graph formed from a cycle of length $k$ by introducing a new vertex adjacent to all vertices in the cycle.) The ideal generated by all tree polynomials is radical $[5$, Theorem 1.1]. It is conjectured, and has been verified experimentally for $n \leq 9$, that the ideal of all tree polynomials is in fact generated by the subset $\left\{\tau_{Q}\right\}$ [5], where $Q$ is a 3 -wheel (equivalently, a 4-clique) in $K_{n}$.

The tree polynomials have integer coefficients, which raises the question of counting their solutions over a finite field. Let $\mathbb{F}_{q}$ be the field with $q$ elements. In this article, we count the solutions of the tree polynomials over $\mathbb{F}_{2}$ and give some generalizations for $q>2$. When the slope variety is considered over $\mathbb{F}_{q}$, the points correspond to drawings, in $\overline{\mathbb{F}}_{q}^{2}$ whose slopes are in $\mathbb{F}_{q}$. These drawings need not be in $\mathbb{F}_{q}^{2}$. If $q=2$, then there is no way to draw $K_{n}$, for $n \geq 3$, so that the vertices have distinct $x$-coordinates. When considered 
over $\mathbb{Z}$, the tree polynomials are in the kernel of the map from $\mathbb{Z}\left[\left\{m_{i, j}\right] \rightarrow \mathbb{Q}\left[\left\{x_{i}, y_{i}, \frac{1}{x_{i}-x_{j}}\right\}\right]\right.$ defined by $m_{i, j} \mapsto \frac{y_{i}-y_{j}}{x_{i}-x_{j}}$.

Theorem 1. Let $n$ be a positive integer and let $\mathbb{F}_{2}\left[K_{n}\right]:=\mathbb{F}_{2}\left[m_{1,2}, \ldots, m_{n-1, n}\right]$. Let $I_{n}$ denote the ideal of $\mathbb{F}_{2}\left[K_{n}\right]$ generated by the tree polynomials of wheel subgraphs of $K_{n}$, and let $J_{n}$ denote the ideal generated by the tree polynomials of $K_{4}$-subgraphs of $K_{n}$ (so $\left.J_{n} \subseteq I_{n}\right)$.

Then the following sets are equinumerous:

1. the zeroes of $I_{n}$, i.e., the points in $\mathbb{F}_{2}^{\left(\begin{array}{c}n \\ 2\end{array}\right)}$ on which all tree polynomials vanish;

2. the zeroes of $J_{n}$, i.e., the points in $\mathbb{F}_{2}^{\left(\begin{array}{c}n \\ 2\end{array}\right)}$ on which all tree polynomials of 3-wheels vanish;

3. complement-reducible graphs (or "cographs") on vertex set $[n]=\{1,2, \ldots, n\}$, that is, graphs on $[n]$ having no induced subgraph isomorphic to a four-vertex path;

4. switching-equivalence classes of graphs on vertex set $[n+1]$ such that no member of the class contains an induced 5-cycle.

We will explain all these combinatorial interpretations below. The following Theorem appears in [8, Exercise 5.40] and is credited to Cameron [2].

Theorem 2. The following sets are equinumerous:

1. switching-equivalence classes of graphs on vertex set $[n+1]$ such that no member of the class contains an induced 5-cycle;

2. series-parallel posets with n labeled vertices;

3. series-parallel networks with $n$ labeled edges.

In this paper, we use the special structure of tree polynomials to prove first the equality of (1), (2) and (3) of Theorem 1 (Proposition 6), and then a bijection between (3) and (4) of Theorem 1 (Proposition 7).

We note that a bijection between unlabeled complement-reducible graphs and unlabeled series-parallel networks was given by Sloane, see sequence A000084 [6]. We have not found in the literature an explicit bijection for the corresponding labeled objects. The numbers of points in $S_{2}\left(K_{1}\right), S_{2}\left(K_{2}\right), \ldots$, are

$$
1,2,8,52,472,5504,78416, \ldots
$$

which is sequence A006351 in [6]. 


\section{Background}

\subsection{Graph theory}

We list some necessary notation here; for a general background on graph theory see [1] or [9]. A graph $G$ is an ordered pair $(V, E)$ of vertices and edges i.e., $V$ is a finite set, and $E$ is a set of 2-subsets of $V$. Two vertices $u, v \in V$ are adjacent if there is an edge $u v \in E$ between them. If $V, E$ are not specified then $V(G)$ is the set of vertices of $G$ and $E(G)$ is the edge set. All graphs in this paper are simple, i.e., they have no loops or multiple edges.

For $U \subseteq V$, the induced subgraph $\left.G\right|_{U}$ of $G$ on $U$, is the graph with vertex set $U$ and edge set $\{u v \in E(G) \mid u, v \in U\}$. The intersection $G \cap H$ of two graphs $G$ and $H$ is the graph with vertex set $V(G) \cap V(H)$ and edge set $E(G) \cap E(H)$. The complement $\bar{G}$ of $G$ is the graph on the same set of vertices as $G$ whose edges are exactly the non-edges of $G$.

Let $K_{n}$ denote the complete graph on $n$ vertices. Let $P_{n}$ denote the path on $n$ vertices, also called the $n$-path. A complement-reducible graph, or cograph, has no induced $P_{4}$. An important fact that we will need is that $G$ is complement-reducible if and only if for every induced subgraph $H \subseteq G$, either $H$ or the complement $\bar{H}$ is disconnected; see [3].

The $k$-wheel $W\left(v_{0} ; v_{1}, \ldots, v_{k}\right)$ is the graph on the vertices $\left\{v_{0}, \ldots, v_{k}\right\}$ and whose edges are $v_{0} v_{1}, \ldots, v_{0} v_{k}, v_{1} v_{2}, \ldots, v_{k-1} v_{k}, v_{k} v_{1}, k \geq 3$. Note that the wheel $W\left(v_{0} ; v_{1}, \ldots, v_{k}\right)$ is invariant up to dihedral permutations of $v_{1}, \ldots, v_{k}$. The vertex $v_{0}$ is called the center; the other vertices are called the spokes. The edges incident to the center are called the radii, and the other edges are chords. Note that a 3-wheel is the complete graph on four vertices.

\subsection{Series-parallel networks}

A network is a graph $G$ with two vertices $s_{G}, t_{G}$ designated as the source and sink, respectively. Two networks $G$ and $H$ can be connected in series or parallel. The series connection $G \oplus H$ is defined by identifying $t_{G}$ with $s_{H}$, and designating $s_{G}$ as the source and $t_{H}$ as the sink. The parallel connection $G+H$ is defined by identifying $s_{G}$ with $s_{H}$ and $t_{G}$ with $t_{H}$.

A series-parallel network is a graph obtained from the following rules:

1. a graph with one edge st is a series-parallel network;

2. if $G$ and $H$ are series-parallel networks, then $G \oplus H$ and $G+H$ are series-parallel networks.

One can define series and parallel connections for posets in a similar fashion; see $[7$, Section 3.2]. Two posets $P$ and $Q$ are connected in series by taking their ordinal sum $P \oplus Q$ : declaring that all elements of $Q$ are larger than all elements of $P$ (or vice versa) leaving all other relations unchanged. The two posets are connected in parallel by taking the disjoint union. A series-parallel poset is a poset built up from single-element posets by series and parallel extensions. 
Let $s(n)$ be the number of labeled series-parallel networks on $n$ vertices. The sequence begins

$$
s(1)=1, \quad s(2)=2, \quad s(3)=8, \quad s(4)=52, \quad s(5)=472, \quad s(6)=5504, \quad \ldots
$$

This is sequence A006351 in the On-Line Encyclopedia of Integer Sequences [6].

\subsection{Switching equivalence}

Let $G$ be a graph on $[n+1]$ and let $X \subseteq[n]$. The switch of $G$ with respect to $X$ is the graph $s_{X}(G)$ on $[n+1]$ whose edges $e$ satisfy one of two conditions:

1. $e \in E(G)$ and either both vertices of $e$ belong to $X$ or neither do;

2. $e \notin E(G)$ and exactly one vertex of $e$ belongs to $X$.

This operation is also referred to as graph switching or Seidel switching [10]. Let $\mathscr{G}_{n+1}$ be the set of graphs on $[n+1]$. Then switching defines an action of $\mathbb{Z}_{2}^{n}$ on $\mathscr{G}_{n+1}$. For $x=\left(x_{1}, \ldots, x_{n}\right) \in \mathbb{Z}_{2}^{n}$, let $X=\left\{i \mid x_{i}=1\right\} \subset[n]$. Then the group action is $x G=s_{X}(G)$. This action is free because $s_{X}(G)=G$ if and only if $X=\emptyset$. The orbits are called switching classes, denoted by $[G]$. To see that each orbit contains exactly one graph in which the vertex $n+1$ is isolated, let $G \in \mathscr{G}_{n+1}$ and let $X=N(n+1)$ be the set of neighbors of $n+1$. Then the graph $s_{X}(G)$ has $n+1$ as an isolated vertex. On the other hand if $X$ is any other subset of $[n]$, then $n+1$ will be adjacent to some vertex of $s_{X}(G)$. The number of switching classes on $[n+1]$ is $s(n)$, the number of labeled series-parallel networks [8, Exercise 5.40(b)], [2].

\subsection{Tree polynomials}

We briefly sketch the basics of graph picture spaces; for more details, see [4].

Definition 3. Let $G=(V, E)$ be a graph. For each $e \in E$, let $m_{e}$ be a variable. For each subset $F \subseteq E$ define

$$
m_{F}=\prod_{f \in F} m_{f}
$$

We regard the square-free monomial $m_{F}$ as corresponding to the spanning subgraph $(V, F)$, and we will often ignore the distinction between the monomial and the graph.

A picture of a graph $G=(V, E)$ is a collection of labeled points and lines in the plane, corresponding to the vertices and edges of $G$, respectively, such that the line $\ell_{e}$ corresponding to an edge $e$ contains both points corresponding to the endpoints of $e$. The vertices have distinct locations. The plane in [4] is taken to be $\mathbb{F}^{2}$ where $\mathbb{F}$ is algebraically closed. Provided that no lines are vertical, each line $\ell_{e}$ has a well-defined slope $m_{e}$, and so each picture determines a slope vector $\left(m_{e}\right)_{e \in E}$. The set of all possible slope vectors is called the slope variety $S(G)$. 
The slope variety is the set of common zeroes of the set of polynomials called tree polynomials, as we now explain. A (rigidity) pseudocircuit is a graph $H$ whose edge set can be partitioned into two spanning trees. A coupled spanning tree of $H$ is a tree whose complement is also a spanning tree; the set of all coupled spanning trees of $H$ is denoted $\mathrm{Cpl}(H)$. For each pseudocircuit $H \subseteq G$, there is a polynomial

$$
\tau_{H}=\sum_{T \in C p l(H)} \epsilon(H, T) m_{T}
$$

that vanishes on the slope variety of $G$; where each $\epsilon(H, T) \in\{1,-1\}$. The sign $\epsilon(H, T)$ arises because the tree polynomial is the determinant of a matrix whose entries are either 0 or of the form $m_{e}-m_{f}$. For a $k$-wheel, the sign is determined by the parity of $k$ and the parity of the number of radii in the coupled spanning tree. Call this polynomial the tree polynomial of $H$.

Because the tree polynomials have integer coefficients, it makes sense to consider these polynomials inside the polynomial ring

$$
\mathbb{F}_{q}[G]:=\mathbb{F}_{q}\left[m_{e} \mid e \in E(G)\right] .
$$

Define the q-slope variety $S_{q}(G)$ to be the zero set of the ideal generated by the tree polynomials of all pseudocircuit subgraphs of $G$. The main concern of this paper is $S_{2}\left(K_{n}\right)$, the set of zeroes of the complete graph over $\mathbb{F}_{2}$.

The most important pseudocircuits are the wheels. The tree polynomial of the wheel $W=W\left(v_{0} ; v_{1}, \ldots, v_{k}\right)$ has the form

$$
\tau_{W}=\underbrace{\prod_{i=1}^{k}\left(m_{0, i}-m_{i, i+1}\right)}_{\tau_{1}}-\underbrace{\prod_{i=1}^{k}\left(m_{0, i}-m_{i-1, i}\right)}_{\tau_{2}}
$$

where $m_{k, k+1}=m_{1, k}[5$, eqn. (6)].

Suppose we draw the wheel $W\left(v_{0} ; v_{1}, \ldots, v_{k}\right)$ with $v_{0}$ in the center and the indices of the spokes increasing as we travel clockwise around the perimeter. Each binomial factor in $\tau_{1}$ is a radius minus the adjacent chord pointing in the clockwise direction, whereas each binomial factor in $\tau_{2}$ is a radius minus the adjacent chord pointing in the counterclockwise direction. Therefore, if we expand the expression (2) for $\tau_{W}$, then the claw subgraph (i.e., the graph consisting of three edges that meet at a point) and the cycle of all the chords each occur twice, and with opposite signs. The only remaining terms are coupled spanning trees, which are obtained by picking a nontrivial subset of radii along with all chords pointing clockwise or counterclockwise, but not both. See Figure 1.

The tree polynomials of all the wheels in $K_{n}$ generate the ideal of tree polynomials of all rigidity pseudocircuits in $K_{n}[5]$. Define ideals $I_{n}, J_{n} \subseteq \mathbb{F}_{2}[G]$ as follows:

$$
\begin{aligned}
& I_{n}=\left(\tau_{W} \mid W \text { is a wheel in } K_{n}\right), \\
& J_{n}=\left(\tau_{Q} \mid Q \subseteq K_{n} \text { is isomorphic to } K_{4}\right) .
\end{aligned}
$$

It was conjectured in [5] that $I_{n}=J_{n}$ when considered as ideals over $\mathbb{C}$. Using the computer algebra system Macaulay this conjecture has been verified for $n \leq 9$ [5]. 


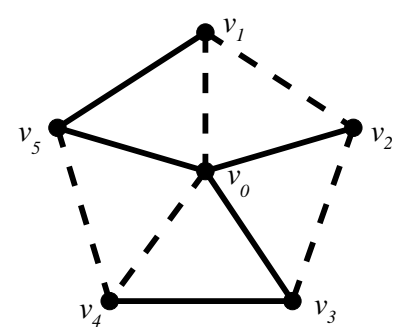

Figure 1: Two complementary coupled spanning trees of a 5-wheel

\section{A bijection between slope vectors and complement- reducible graphs}

In this section we count the points of $S_{2}\left(K_{n}\right)$, the slope variety of $K_{n}$ over $\mathbb{F}_{2}$. The points of $\mathbb{F}_{2}^{\left(\begin{array}{c}n \\ 2\end{array}\right)}$ have their coordinates indexed by the edges of $K_{n}$ and have value either 0 or 1 , which motivates the following notation:

Definition 4. Let $a=\left(a_{1,2}, a_{1,3}, \ldots, a_{n-1, n}\right) \in \mathbb{F}_{2}^{\left(\begin{array}{c}n \\ 2\end{array}\right)}$. We define the graph $G_{a}$ to be the graph on $[n]$ with edge set $E\left(G_{a}\right)=\left\{i j \mid a_{i, j}=1\right\}$.

Proposition 5. Let $W=W\left(v_{0} ; v_{1}, \ldots, v_{k}\right)$ be a wheel and $a \in \mathbb{F}_{2}^{\left(\begin{array}{c}n \\ 2\end{array}\right)}$. Then $\tau_{W}(a) \neq 0$ if and only if $H_{a}:=G_{a} \cap W$ is a coupled spanning tree of $W$.

Proof. $(\Leftarrow)$ Suppose that $H_{a}$ is a coupled spanning tree of $W$. When $\tau_{W}$ is written in the form of equation (1), over $\mathbb{F}_{2}$, it is the sum of all coupled spanning trees of $W$. Evaluating $\tau_{W}$ at $a$ gives exactly one non-zero term, hence $\tau_{W}(a) \neq 0$.

$(\Rightarrow)$ Suppose $\tau_{W}(a) \neq 0$. First we show that $H_{a}$ is a spanning tree of $W$. Over $\mathbb{F}_{2}$ exactly one of $\tau_{1}(a)$ or $\tau_{2}(a)$ has value 1 , say $\tau_{1}(a)=1$. Each binomial factor of $\tau_{1}(a)$ must contain exactly one variable with value 1 . Therefore, $H_{a}$ contains exactly $k$ edges, which is the number of edges of a spanning tree of $W$. In order to show that $H_{a}$ is a spanning tree it is enough to show that it is acyclic. If $H_{a}$ contains a cycle $C$, then either there exist $i$ and $j, 1 \leq i<j \leq k$, such that $v_{0} v_{i}, v_{i} v_{i+1}, v_{j-1} v_{j}, v_{0} v_{j} \in E(C)$, or $C$ is the set of chords of $W$. In the first case, both terms $\tau_{1}(a)$ and $\tau_{2}(a)$ have value 0 because $m_{0 i}-m_{i(i+1)}$ is a factor of $\tau_{1}$ and $m_{0 j}-m_{(j-1) j}$ is a factor of $\tau_{2}$. In the second case, formula (2) will be

$$
\tau_{W}(a)=\prod_{i=1}^{k}\left(a_{0 i}-1\right)-\prod_{i=1}^{k}\left(a_{0 i}-1\right)=0 .
$$

Now we show that $H_{a}$ is in fact a coupled spanning tree of $W$. Define $\bar{a} \in \mathbb{F}_{2}^{\left(\begin{array}{c}n \\ 2\end{array}\right)}$ by $\overline{a_{i j}}=1-a_{i j}$ for all $1 \leq i<j \leq n$. Therefore, $G_{\bar{a}}=\overline{G_{a}}$ is the complement of $G_{a}$. If $\tau_{W}(a) \neq 0$ then $\tau_{W}(\bar{a}) \neq 0$ because each binomial factor of $\tau_{i}(\bar{a})$ will have the same value 
as in $\tau_{i}(a)$, for $i=1,2$. Therefore $H_{\bar{a}}=\overline{H_{a}} \cap W$ is a spanning tree of $W$, hence $H_{a}$ is a coupled spanning tree of $W$.

The following proposition gives additional evidence to suggest that $I_{n}$ and $J_{n}$ are equal, but whether or not they are equal is still unknown.

Proposition 6. Let $a \in \mathbb{F}_{2}^{\left(\begin{array}{l}n \\ 2\end{array}\right)}$. The following are equivalent:

1. a is a zero of $I_{n}$;

2. $a$ is a zero of $J_{n}$;

3. $G_{a}$ is a complement-reducible graph.

Proof. $(1 \Rightarrow 2)$ This implication follows from the containment $J_{n} \subseteq I_{n}$.

$(2 \Rightarrow 3)$ Suppose $a \in \mathbb{F}_{2}^{\left(\begin{array}{l}n \\ 2\end{array}\right)}$ is a zero of $J_{n}$. By Proposition 5 , if $W \subseteq K_{n}$ is any 3-wheel (and hence isomorphic to $K_{4}$ ), then $G_{a} \cap W$ is not a coupled spanning tree of $W$. Since every coupled spanning tree of $K_{4}$ is isomorphic to $P_{4}$ (the only spanning trees of $K_{4}$ are isomorphic to $P_{4}$ or the three edge claw), $G_{a}$ does not contain an induced $P_{4}$.

$(3 \Rightarrow 1)$ Let $a \in \mathbb{F}_{2}^{\left(\begin{array}{c}n \\ 2\end{array}\right)}$ be such that $G_{a}$ is a complement-reducible graph. Let $W \subseteq K_{n}$ be a wheel with $V=V(W)$. Either $\left.G_{a}\right|_{V}$ or $\left.\bar{G}_{a}\right|_{V}$ is disconnected, because $G_{a}$ is a complement-reducible graph. Therefore either $G_{a} \cap W$ or $G_{\bar{a}} \cap W$ is disconnected. Since these two graphs are complementary subgraphs of $W$, neither one is a coupled spanning tree. Therefore, by Proposition $5, \tau_{W}(a)=0$ for every wheel $W \subseteq K_{n}$.

\section{A bijection between complement-reducible graphs and switching classes}

In this section, we establish a bijection (Proposition 7) between the set of graphs on $n$ labeled vertices with an induced $P_{4}$, and the switching classes on $n+1$ labeled vertices containing a graph with an induced 5-cycle. Recall from Section 2.3 that each switching class contains exactly one graph in which the vertex $n+1$ is isolated. Therefore the bijection from the set of graphs on $[n]$ to the switching classes on $[n+1]$ is given by sending $G \subseteq K_{n}$ to $[G]$, the orbit containing $G$.

Proposition 7 . Let the additive group $\mathbb{Z}_{2}^{n}$ act on $\mathscr{G}_{n+1}$ by switching as described in Section 2.3. Then:

1. If $G \in \mathscr{G}_{n+1}$ has an induced 5-cycle, then every $H \in[G]$ has an induced 4-path.

2. If $G \in \mathscr{G}_{n}$ has an induced 4-path, then, regarding $G$ as a graph on $[n+1]$ by introducing $n+1$ as an isolated vertex, there is an $H \in \mathscr{G}_{n+1}$ such that $G \in[H]$ and $H$ has an induced 5-cycle. 

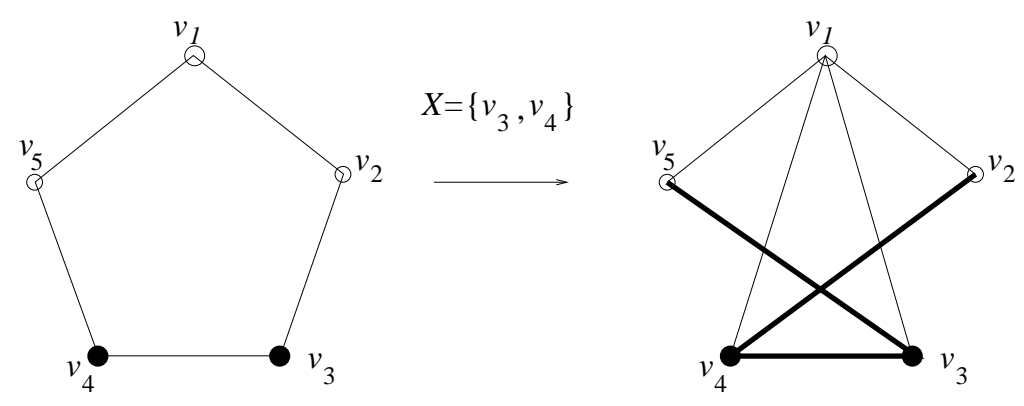

Figure 2: The action with $X=\left\{v_{3}, v_{4}\right\}$
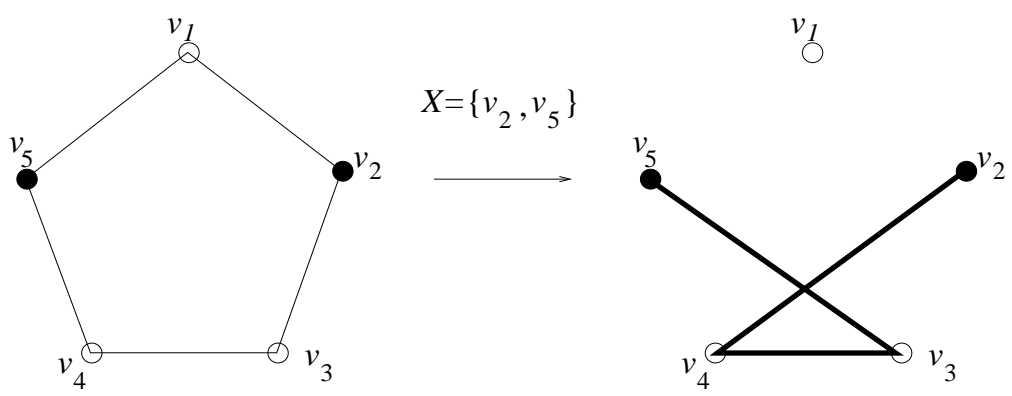

Figure 3: The action with $X=\left\{v_{2}, v_{5}\right\}$

Proof. (1) Let $G \in \mathscr{G}_{n+1}$ have an induced 5-cycle $C=\left\{v_{1}, \ldots, v_{5}\right\}$, and let $X \subseteq[n]$. If $|V(C) \cap X|<2$, then four of the vertices, say $U=\left\{v_{1}, v_{2}, v_{3}, v_{4}\right\}$, are in $[n] \backslash X$. Switching by $X$ does not affect the induced subgraph on $U$. Similarly, if $|V(C) \cap X|>3$, then $\left(s_{X}(G) \mid U\right) \cong P_{4}$.

Suppose $|V(C) \cap X|=2$. Without loss of generality we may assume either $X=\left\{v_{2}, v_{5}\right\}$ or $X=\left\{v_{3}, v_{4}\right\}$. In both cases $v_{5} v_{3} v_{4} v_{2}$ is an induced 4-path in $s_{X}(G)$, as shown in the figure. If $|V(C) \cap X|=3$, then $|V(C) \cap([n+1] \backslash X)|=2$. The same results as above will hold for this case, therefore $s_{X}(G)$ has an induced $P_{4}$.

(2) Suppose $v_{5} v_{3} v_{4} v_{2}$ is an induced $P_{4}$ in $G \subseteq K_{n}$ and $X=\left\{v_{2}, v_{5}\right\}$. Then $s_{X}(G)$ has the induced 5-cycle $C=\left\{v_{1}, \ldots, v_{5}\right\}$ with $v_{1}=n+1$.

\section{Counting points over other finite fields}

It is natural to ask whether these techniques can be extended to enumerate points of the slope variety $S_{q}\left(K_{n}\right)$ over $\mathbb{F}_{q}$. This problem appears to be difficult, because the zeroes of a tree polynomial over an arbitrary field do not seem to admit a uniform graph-theoretic description as they do over $\mathbb{F}_{2}$. In this section, we describe some partial progress in this direction, and explicitly work out the simplest nontrivial case $(n=4, q=3)$ to illustrate the kinds of difficulties involved.

A point in $\mathbb{F}_{q}^{\left(\begin{array}{c}n \\ 2\end{array}\right)}$ corresponds to an $\mathbb{F}_{q}$-weighted $K_{n}$, that is, a copy of $K_{n}$ whose edges 


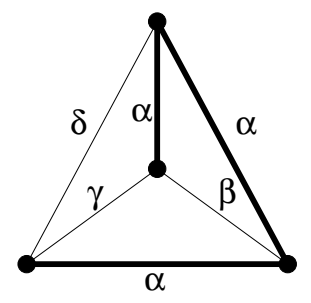

Figure 4: A weight induced $P_{4}$

are assigned weights in $\mathbb{F}_{q}$. For $a \in \mathbb{F}_{q}^{\left(\begin{array}{c}n \\ 2\end{array}\right)}$ define $G_{a}$ to be the $\mathbb{F}_{q}$-weighted $K_{n}$ where edge $i j$ is given weight $a_{i j}$. We say that $G_{a}$ has a weight-induced subgraph $H$ if there is some value $\alpha \in \mathbb{F}_{q}$ such that

$$
E(H)=\left\{e \in E\left(K_{n}\right) \mid a_{e}=\alpha\right\} .
$$

One possible approach to generalizing the previous results would be to define a $q$ analogue to switching. Let the additive group $\mathbb{F}_{q}^{n}$ act on $\mathbb{F}_{q}^{\left(\begin{array}{c}n \\ 2\end{array}\right)}$ by

$$
\left.\left(\left(x_{1}, \ldots, x_{n}\right) \cdot a\right)\right)_{i j}=\left(a_{i j}+x_{i}+x_{j}\right) .
$$

If $q=2$, then this is exactly the switching action described in Section 2.3. Note that this is not the same definition of $q$-switching given by Zaslavsky [10]. The present definition seems more likely to be relevant in the context of slopes because it does not rely on an orientation of the edges. (Recall that the weight of an edge is the slope of the corresponding line segment in a picture of $K_{n}$; the slope does not depend on the direction in which way the edge is traversed.) One would hope to generalize the $q=2$ case by describing the points of $S_{q}\left(K_{n}\right)$ in terms of forbidden weight-induced subgraphs. It is not clear how to generalize the definition over an arbitrary field, or what the forbidden weight-induced subgraphs should be. However, some facts do carry over to the setting of an arbitrary finite field.

Proposition 8. Let $W=W\left(v_{0} ; v_{1}, v_{2}, v_{3}\right)$ be a 3-wheel, and let $a \in \mathbb{F}_{q}^{\left(\begin{array}{l}4 \\ 2\end{array}\right)}$ be a point whose coordinates correspond to assigning weights to the edges of $W$. Then:

1. If $G_{a}$ has a weight-induced $P_{4}$, then a is not a zero of $\tau_{W}$.

2. If $G_{a}$ has a weight-induced claw (that is, a star with three edges), then a is a zero of $\tau_{W}$.

3. If $G_{a}$ has a weight-induced cycle, then a is a zero of $\tau_{W}$.

Proof. (1) Suppose that $G_{a}$ has a weight-induced $P_{4}$. The induced subgraph on the vertices of that $P_{4}$ can be drawn as in Figure 4 . where $\alpha, \beta, \gamma, \delta \in \mathbb{F}_{q}$, and $\alpha$ does not equal any of the other values. Then,

$$
\tau_{W}(a)=(\alpha-\alpha)(\beta-\alpha)(\gamma-\delta)-(\alpha-\delta)(\beta-\alpha)(\gamma-\alpha) \neq 0 .
$$


(2) Suppose that $G_{a}$ has a weight-induced claw, whose edges have the weight $\alpha \in \mathbb{F}_{q}$. If we draw $W$ so that the center of the claw is the center of the wheel, then

$$
\tau_{Q}(a)=(\alpha-\beta)(\alpha-\gamma)(\alpha-\delta)-(\alpha-\gamma)(\alpha-\delta)(\alpha-\beta)=0,
$$

for some $\beta, \gamma, \delta \in \mathbb{F}_{q}$.

(3) Suppose that $G_{a}$ has a weight-induced cycle $C$. The graph $W$ can be drawn so that $C$ contains the vertex $v_{0}$. Then, for some $1 \leq i<j \leq 3$, the edges $v_{0} v_{i}, v_{i} v_{i+1}, v_{0} v_{j}$, $v_{j} v_{j-1}$ all have the same weight $\alpha$. (Note that if the cycle is a 3-cycle then $v_{j-1}=v_{i}$.) Then both $\tau_{1}(a)$ and $\tau_{2}(a)$ contain the factor $\alpha-\alpha$, so $\tau_{W}(a)=0$.

Corollary 9. Let $a \in \mathbb{F}_{q}^{\left(\begin{array}{c}n \\ 2\end{array}\right)}$. If $G_{a}$ contains a weight-induced $P_{4}$, then a is not a zero of $I_{n}$ over $\mathbb{F}_{q}$. Conversely, if every 4-clique of $G_{a}$ contains a weight-induced cycle or a weight-induced claw, then a is a zero of $J_{n}$ over $\mathbb{F}_{q}$.

Example 10. Let $W=W\left(v_{0} ; v_{1}, v_{2}, v_{3}\right)$ be a 3 -wheel. We use Proposition 8 to count the number of zeroes of $\tau_{W}$ over $\mathbb{F}_{3}$.

If some value occurs at least four times in $a$, then $\tau_{W}(a)=0$ because $G_{a}$ has a weightinduced cycle. If some value $\alpha$ occurs exactly three times in $a$, then $\tau_{W}(a) \neq 0$ if and only if the weight-induced graph on $\alpha$ is a 4-path. The cases where each value of $a$ occurs two times are not covered by Proposition 8, so we must consider them separately. For distinct $\alpha, \beta, \gamma \in \mathbb{F}_{3}$ there are three possibilities, up to a relabeling of the vertices; see Figure 5 .
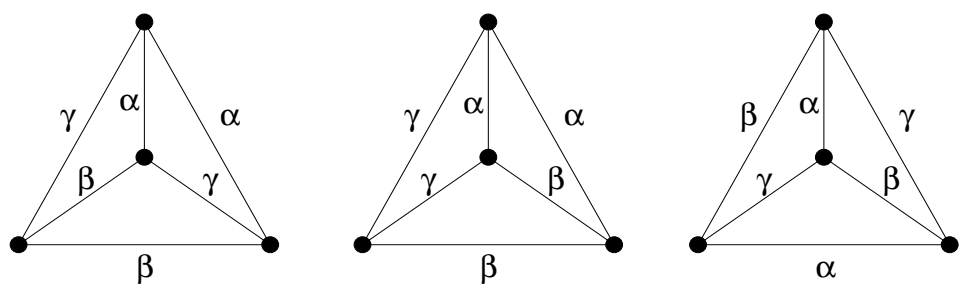

Figure 5: The first weight corresponds to a zero of $\tau_{W}$, but the second two do not.

Define the type of $a \in \mathbb{F}_{q}^{d}$ to be the partition whose parts are the numbers of occurrences of each element of $\mathbb{F}_{q}$ among the entries of $a$. Some simple counting gives the following table:

\begin{tabular}{c|c|c} 
Type & Number of zeroes & Number of non-zeroes \\
\hline$(6)$ & 3 & 0 \\
$(5,1)$ & 36 & 0 \\
$(4,2)$ & 90 & 0 \\
$(4,1,1)$ & 90 & 0 \\
$(3,3)$ & 24 & 36 \\
$(3,2,1)$ & 144 & 216 \\
$(2,2,2)$ & 36 & 54 \\
\hline Total & 423 & 306
\end{tabular}


If $q>3$, then there are more cases to check which are not covered by Proposition 8 . Using the computer algebra software Maple, one can check that over $\mathbb{F}_{3}$ the number of zeroes of $I_{4}$ and $I_{5}$ are 423 and 9243, respectively. Over $\mathbb{F}_{5}$ the numbers are 4909, 262645, respectively. It is not clear what combinatorial structure (analogous to complementreducible graphs) might count these points; for instance, these numbers do not appear in the Encyclopedia of Integer Sequences [6].

\section{References}

[1] Béla Bollobás, Modern graph theory, Graduate Texts in Mathematics, vol. 184, Springr-Verlag, New York, 1998. MR MR1633290 (99h:05001)

[2] Peter J. Cameron, Counting two-graphs related to trees, Electron. J. Combin. 2 (1995), Research Paper 4, approx. 8 pp. (electronic). MR MR1312733 (95j:05112)

[3] D. G. Corneil, H. Lerchs, and L. Stewart Burlingham, Complement reducible graphs, Discrete Appl. Math. 3 (1981), no. 3, 163-174. MR MR619603 (84d:05137)

[4] Jeremy L. Martin, Geometry of graph varieties, Trans. Amer. Math. Soc. 355 (2003), no. 10, 4151-4169 (electronic). MR MR1990580 (2005b:05074)

[5] - The slopes determined by $n$ points in the plane, Duke Math. J. 131 (2006), no. 1, 119-165. MR MR2219238 (2007e:05041)

[6] N. J. A. Sloane, The On-Line Encyclopedia of Integer Sequences, http://oeis.org, 2010.

[7] Richard P. Stanley, Enumerative combinatorics. Vol. 1, Cambridge Studies in Advanced Mathematics, vol. 49, Cambridge University Press, Cambridge, 1997, With a foreword by Gian-Carlo Rota, Corrected reprint of the 1986 original. MR MR1442260 (98a:05001)

[8] _ Enumerative combinatorics. Vol. 2, Cambridge Studies in Advanced Mathematics, vol. 62, Cambridge University Press, Cambridge, 1999, With a foreword by Gian-Carlo Rota and appendix 1 by Sergey Fomin. MR MR1676282 (2000k:05026)

[9] Douglas B. West, Introduction to graph theory, Prentice Hall Inc., Upper Saddle River, NJ, 1996. MR MR1367739 (96i:05001)

[10] Thomas Zaslavsky, Glossary of signed and gain graphs and allied areas, Electron. J. Combin. 5 (1998), Dynamic Surveys 9, 41 pp. (electronic). MR 1744870 (2000m:05001b) 